\title{
Historia de la educación, género y perspectivas docentes
}

\author{
History of education, gender and teachers' perspectives
}

Laura Rangel Bernal ${ }^{1}$

\section{Resumen}

Esta reseña describe y comenta el contenido del libro "Historia de la educación, género y perspectivas docentes", coordinado por Norma Gutiérrez Hernández, Beatriz Marisol García Sandoval, María del Refugio Magallanes Delgado e Irma Faviola Castillo Ruiz. El libro está conformado por 17 capítulos. En su redacción participaron 31 autores y autoras procedentes de diferentes universidades. Se trata de una labor conjunta, con enfoque multidisciplinar. En sus páginas se integran aportaciones de áreas como la historia, la literatura, la lingüística, las artes visuales, las ciencias de la salud y las matemáticas. Los capítulos referentes a la historia de la educación constituyen un aporte significativo a la historiografía educativa de la región. Además, contribuyen al análisis de la intersección entre la historia y los estudios de género, dado que abordan problemáticas relativas a la educación de las mujeres en los siglos XIX y XX.

\section{Palabras clave: historiografía, desarrollo profesional docente, estudios de género}

\section{Abstract}

This review describes and comments on the content of the book History of education, gender and teachers' perspectives, which was coordinated by por Norma Gutiérrez Hernández, Beatriz Marisol García Sandoval, María del Refugio Magallanes Delgado e Irma Faviola Castillo Ruiz. The book contains 17 chapters which were written by a total of 31 authors from different universities. Its publication is the product of a collaborative effort with a multidisciplinary approach. Contributions of various fields appear in its pages, like history, literature, linguistics, the visual arts, health sciences and mathematics, to name a few. The chapters that talk about the history of education

\footnotetext{
${ }^{1}$ Unidad Académica de Docencia Superior de la Universidad Autónoma de Zacatecas.

Correo electrónico: laura.rangelb@gmail.com
} 
constitute a significant contribution to the field of historiography in the region. Additionally, they bring in elements to the analysis of the intersection between history and gender studies, given that they study problems related to the education of women during the nineteenth and twentieth centuries.

\section{Keywords: historiography, teachers' professional development, gender studies}

\section{Reseña del libro: Historia de la educación, género y perspectivas docentes}

El libro Historia de la educación, género y perspectivas docentes sale a la luz este año 2021, en medio de condiciones inusitadas y que pueden entenderse de varias formas como adversas para la producción académica, dadas las múltiples restricciones que se han impuesto como parte de las medidas para limitar el avance y las afectaciones de la pandemia que aún no termina. Es por ello que el trabajo de quienes participaron en la coordinación de este libro: es doblemente meritorio, primero por el producto que entregan y, en segundo lugar, por el esfuerzo que implicó su producción.

El libro fue publicado por la Universidad Autónoma de Zacatecas y está conformado por 17 capítulos, escritos por un total de 31 autores y autoras, en su mayoría en colaboración. Se trata de una labor conjunta, con un enfoque multidisciplinar, pues en la redacción participaron investigadoras e investigadores de diferentes instituciones académicas de nivel superior como la Universidad Autónoma de Zacatecas, la Universidad Pedagógica Nacional Unidad Zacatecas, el Centro de Actualización del Magisterio de Zacatecas, la Universidad Autónoma de Querétaro y la Universidad de Gotemburgo, Suecia. Todos ellos y ellas investigan en torno a temas vinculados a la educación desde diferentes áreas del conocimiento como son la historia, la pedagogía, la psicología, la literatura, la lingüística, las artes visuales, las ciencias de la salud, las ciencias políticas y las matemáticas. Los aportes tanto teóricos como metodológicos de todas estas disciplinas se plasman en los capítulos que conforman el libro, brindando un mosaico que se complementa con hallazgos empíricos, así como con las reflexiones e interpretaciones críticas que hacen los propios autores y autoras sobre los temas que analizan.

Los capítulos se desarrollan en torno a tres ejes temáticos interconectados: la historia de la educación, el género y las perspectivas docentes. Los trabajos que se integran el primer eje permiten comprender las políticas y realidades educativas a nivel regional desde una perspectiva histórica, pues nos hablan del sinuoso y difícil trayecto que se tuvo que seguir, una vez consumada la independencia, para poder establecer las bases del sistema educativo 
nacional y asegurar su bien funcionamiento en periodos de agitación que se abarcaron prácticamente todo el siglo XIX y se extendieron hasta entrado el siglo XX.

El tema de género se aborda desde la historia de la educación de las mujeres en el siglo XIX y principios del XX. Se discurre sobre la presencia social de las mujeres en México; sobre los avances que se han dado en este sentido y de lo que falta por hacer en la actualidad en diferentes ámbitos: en lo educativo como maestras y alumnas; en el literario, como escritoras y creadoras; en la historia de los conflictos armados y en el imaginario social, como participantes activas de la Revolución Mexicana. En conjunto, estos trabajos, constituyen un aporte significativo a la historiografía educativa de la región centro-norte, además de que contribuyen al análisis de la intersección entre la historia y el género, toda vez que abordar problemáticas relativas a la educación de las mujeres en los siglos XIX y XX.

El eje de las perspectivas docentes se aborda desde la actualidad mediante el estudio de problemas educativos acuciantes y de suma relevancia por sus efectos sociales, como son la deserción y el fracaso escolar, la alimentación saludable en la escuela y el desarrollo de posgrados profesionalizantes orientados a la docencia. Se discute también sobre los intentos por reconfigurar la imagen docente para recuperar su función como agentes sociales de cambio.

En el primer capítulo titulado "La educación en la intendencia de Zacatecas. El artículo 28 de la Ordenanza para la división en cuarteles como beneficio para los comunes. 17961810", Jesús Domínguez Cardiel, Edgar Fernández Álvarez y Marcela Alba Santoyo analizan los elementos modernizadores y disposiciones de corte ilustrado presentes en la Ordenanza de Intendentes de 1786 y en la Ordenanza para la división en cuarteles de la ciudad de Zacatecas. Con base en la evidencia histórica analizada, concluyen que estos documentos legales, que formaron parte de las reformas borbónicas aplicadas en estos territorios, propiciaron la mejora de las condiciones educativas en Zacatecas en este periodo.

Oliva Solís Hernández es la autora del capítulo 2, "El papel de las mujeres en la educación en Querétaro, 1824-1835”. En él, describe las condiciones en las que se educó a las mujeres en el estado de Querétaro durante la Primera República Federal, las cuales fueron mayoritariamente adversas, lo que ocasionó que las niñas escolarizadas fueran una minoría. Aun así, a partir de la década de 1830, algunas mujeres instruidas empezaron a trabajar como preceptoras, con lo cual comenzaron a tener presencia en el espacio educativo y una participación más activa en el contexto social más amplio.

El capítulo 3 lleva por título "Francisco Larroyo y la enseñanza libre en México, 1808-1857". Sus autores José Luis Acevedo Hurtado, Mariana Perea Frausto y Carlos Alberto Pinales Salas analizan de manera detallada el libro Historia comparada de la educación en México (1976), del destacado autor zacatecano Francisco Larroyo. En su análisis, retoman las principales aportaciones que hizo este personaje para establecer una periodización que ha 
servido como modelo para estudiar la historia de la educación en México desde la primera mitad del siglo XX. Además, señalan, con base en los recientes aportes de la historiografía local y regional, temas que quedaron pendientes por estudiar en el periodo que comprende este libro.

El cuarto capítulo intitulado "El Boletín de Instrucción Primaria. Publicación pedagógica de Zacatecas a finales del siglo XIX y principios del XX", es producto de la colaboración entre Norma Gutiérrez Hernández, Beatriz Marisol García Sandoval, María del Refugio Magallanes Delgado e Irma Faviola Castillo Ruiz. En él, las autoras analizan las funciones que tuvieron las publicaciones pedagógicas dirigidas al profesorado zacatecano a principios del siglo XX, tomando el caso específico del Boletín de Instrucción Pública que se constituyó como un instrumento para la formación docente en el estado, particularmente para quienes no contaban con estudios normales y que, en este periodo, fueron la mayoría.

Por su parte, Angélica Ortega Ramírez, en el capítulo 5: "La enseñanza-aprendizaje del dibujo en la Escuela Nacional de Bellas Artes (1900-1914)", describe la forma en la que se enseñó el dibujo en esta reconocida institución de la que egresaron artistas gráficos muy renombrados de principios del siglo pasado. Mediante el análisis del acervo de piezas de dibujo y estampa que se usaban como recursos didácticos, la autora reconstruye los métodos utilizados por los maestros de la academia. Analiza, además, los cambios que se dieron para actualizar dichos métodos. Estos cambios no fueron aceptados con agrado por todos los profesores quienes se resistieron a adoptarlos; situación a la que se sumó el antagonismo existente entre algunos de ellos, por lo que se concluye que el proceso fue difícil por los obstáculos que se presentaron.

Rubén Darío Núñez Altamirano y María del Carmen Loera, en el capítulo 6, "Rebeldía sin representación social: las mujeres en los inicios del siglo XX en México, 1900-1920", analizan la construcción social de los roles de género con base en la participación que tuvieron las mujeres en los ámbitos laboral y militar en una época en la que prevalecía, en el imaginario social, la idea de la mujer vinculada al matrimonio, la familia y las tareas domésticas, siendo el hogar el espacio donde debía desenvolverse. Concluyen que el cambio en estos roles fue un proceso lento, pero llegó a dar resultados en el empoderamiento que obtuvieron algunas mujeres a través de su participación en movimientos sociales y políticos relevantes de la época como fueron las huelgas y protestas y los clubes anti-reeleccionistas.

En el séptimo capítulo, "Corridos, mujeres y revolución. Música para la didáctica y la docencia de la historia", María del Carmen Tatay Fernández y Beatriz Elena Muñoz Serna valoran el uso de la música popular como una estrategia didáctica dirigida a la enseñanza y el aprendizaje de la historia de la Revolución Mexicana. Mediante esta propuesta, que compila una cantidad considerable de corridos que hablan sobre las mujeres protagonistas de este conflicto armado, buscan no solo contribuir a la metodología de la enseñanza de la 
historia, sino también a recuperar las experiencias de estas mujeres y revalorar su papel en la historia de nuestro país.

Cecilia Aguilera Sánchez, quien es autora del octavo capítulo: "Casa, familia y lecturas para mujeres: elementos básicos para una 'reina del hogar', analiza la función y los elementos ideológicos presentes en los textos compilados por la escritora chilena Gabriela Mistral en el libro Lecturas para mujeres (2017), que formó parte del proyecto del entonces secretario de Educación, José Vasconcelos. Estos textos contenían las lecciones morales que se consideraba necesario que asimilara la población femenina, dada su condición de madres, esposas y colaboradoras en la formación de ciudadanos. Para su análisis Cecilia Aguilera los clasifica en temáticas referentes a la casa, la familia, la maternidad, la nación y la patria, el trabajo, y a elementos vinculados con la naturaleza y lo espiritual. Concluye que es evidente que el "ideal femenino" de la época permeó a la selección de estas lecturas.

En "Las leyes de educación primaria en Zacatecas de 1920 a 1940. Entre el tintero y la realidad", noveno capítulo de este libro, Edgar Fernández Álvarez y Juan Manuel Muñoz Hurtado examinan las condiciones en las que se expidieron las leyes educativas de 1921 y 1930, junto con las reformas hechas en 1938 y los resultados de su implementación en este estado, llegando a la conclusión de que, a pesar de que en ellas se establecía la obligatoriedad de la enseñanza primaria, al haber un déficit de escuelas, sobre todo en zonas rurales, una gran parte de la población infantil se quedó al margen de la escolarización. De igual modo, dadas las condiciones sociales de la época, que se vio marcada por el conflicto cristero, se enfatizó el precepto de obligatoriedad en detrimento de la laicidad establecida en la Constitución de 1917.

Mónica Muñoz Muñoz, Estela Galván Cabral y Valeria Moncada León, presentan el décimo capítulo que lleva por título "Investigaciones sobre lengua y género en la Unidad Académica de Letras de la UAZ. Cambio teórico y actitudinal”. En él discurren sobre el actual debate en torno al uso del lenguaje inclusivo que se propone como una alternativa al androcentrismo lingüístico. Si bien su uso es rechazado por instancias internacionales como la Real Academia de la Lengua (RAE), mediante un estudio de caso, las autoras encuentran que personas jóvenes, entre las que se encuentran los estudiantes de la Licenciatura en Letras de la UAZ, manifiestan disposición y apertura hacia la adopción del lenguaje incluso en tanto que reconocen las limitaciones y sesgos del paradigma gramatical y formalista prevalente en la RAE.

Por otro lado, Gabriela Cortez Pérez en "Los referentes literarios de autoría femenina en la UAZ: una aplicación de la disponibilidad léxica", presenta un estudio en el que también participan estudiantes de la UAZ, no solo de la licenciatura en Letras, sino también de otras carreras, para indagar sobre sus referentes literarios y, de este modo, determinar el lugar que ocupan en ellos las escritoras como creadoras de obras literarias. Al respecto encontró que 
son más frecuentes las referencias a autores de sexo masculino, lo cual habla de la necesidad de incorporar en mayor medida obras escritas por mujeres a planes y programas educativos de modo que la literatura deje de ser concebida como un ámbito mayoritariamente masculino.

Cynthia Ivett Campos Ramos y Rosaura Olivia Medina Larios reflexionan, en el capítulo 12: "Desayuno y lonche saludable", sobre la formación de hábitos saludables de alimentación desde la infancia y sobre la importancia de que el alumnado de preescolar pueda consumir alimentos que satisfagan sus necesidades nutrimentales dentro de las horas de clase. Señalan que, para lograr estos propósitos, es necesario desarrollar estrategias en las que se haga partícipes a padres y madres de familia, así como al personal docente, con la finalidad de organizarse para realizar las tareas de preparación de alimentos, lo cual no es sencillo, dadas las limitaciones de tiempo, recursos económicos y de educación nutrimental que presenta la población zacatecana.

"La imagen del y la docente como perspectiva por elevar la calidad en la educación" es el título del décimo tercer capítulo escrito por María Magdalena López Espinosa, quien reflexiona sobre la transformación que ha tenido la imagen pública de los docentes pues esta profesión se ha devaluado en las últimas décadas y perdido el prestigio que alguna vez tuvo. Ello ha impactado en el ejercicio de la labor docente en las aulas. Al respecto, la autora plantea la necesidad de recuperar el papel social que tuvieron los maestros y maestras durante la primera mitad del siglo XX, a fin de fortalecer su imagen y que vuelva a inspirar el respeto que en otras épocas se le confirió.

Brenda Solis Murillo y Amairani Yisel Cardoso Franco presentan "La deserción escolar en la Telesecundaria "Benito Juárez" de La Comunidad de los Ángeles, Fresnillo, Zacatecas", capítulo en el que analizan los factores que inciden en la deserción escolar, siendo que este fenómeno que aqueja de manera más notoria a la población adolescente que habita en el espacio rural. Concluyen que los factores que influyen en mayor medida a la decisión de dejar la escuela son de tipo familiar, económico, laboral y educativo, dado que en el contexto que viven, las y los jóvenes se encuentran en la disyuntiva de seguir estudiando o salir a trabajar para poder aportar a la economía familiar, siendo esta última opción la que consideran más viable.

César Carranza Rodríguez en el capítulo titulado "El ciclo del fracaso escolar en la educación media superior. Un acercamiento a la evaluación de la preparatoria de la Universidad Autónoma de Zacatecas", examina, con base en la información empírica que obtuvo mediante la realización de un estudio de caso, lo que denomina el ciclo del fracaso escolar. Para ello, revisa cada una de las etapas que componen dicho ciclo que son: la reprobación, 
la repitencia, el rezago, el abandono y la deserción. Propone como estrategia la impartición de un curso de regularización que permita al alumnado aprobar, lo más pronto posible, las materias reprobadas y con ello evitar que se repita este ciclo.

Fredrik Olsson en el capítulo 16, "¿Pedagogías sostenibles? Apuntes de un enfoque decolonial de enseñanza-aprendizaje en la educación superior", discurre sobre la existencia de la universidad occidental como institución colonizada, dado su origen europeo, y enfatiza la necesidad de decolonizarla mediante el planteamiento de alternativas pedagógicas que integren el diálogo transdisciplinar y transcultural. Concluye, con base en las propuestas que se han hecho en esta materia en las últimas décadas, que la tarea no será fácil y que "los procesos serán necesariamente contextuales y el resultado de un diálogo abierto desde abajo (p. 214).

José Iván Flores, Carolina Carrillo García y Eduardo Carlos Briceño Solís, cierran este libro con su texto "Caracterización de proyectos de desarrollo profesional en la Maestría en Matemática Educativa de la UAZ". En el reflexionan sobre lo que implica la creación de posgrados profesionalizantes en términos de las características de los trabajos de investigación que realiza el alumnado para obtener el grado. Concluyen, con la base en la información que obtuvieron mediante la aplicación de un instrumento de Análisis de contenido, que los proyectos de desarrollo profesional de esta maestría están fuertemente arraigados a la práctica docente de su alumnado, de modo que esto impacta positivamente en su formación profesional y, en consecuencia, en los estudiantes a quienes imparten clases.

Por último, quisiera resaltar que este libro es producto del trabajo conjunto de las coordinadoras y de un nutrido grupo de investigadoras e investigadores; varios de ellos y ellas noveles, pues están dando sus primeros pasos en el ámbito de la investigación educativa o sobre temas relativos a la educación. En ello radica otro mérito de la obra, que es la apertura de espacios a nuevas voces para que participen activamente en las tareas de producir y difundir textos académicos, con lo cual se abona a la producción del conocimiento en el área de la investigación educativa, las Ciencias Sociales y las Humanidades. 


\section{Referencias bibliográficas}

Larroyo, Francisco (1976), Historia comparada de la educación en México, Porrúa, México.

Mistral, Gabriela (2017), Lecturas para mujeres, Ediciones Porrúa, México.

\section{Referencias electrónicas}

Gutiérrez, Norma, Beatriz Marisol García, María del Refugio Magallanes e Irma Faviola Castillo (2021), Historia de la educación, género y perspectivas docentes, Universidad Autónoma de Zacatecas/Astra Ediciones, México. Disponible en: https://medpd.uaz.edu.mx/ 